\title{
Contribution of IVIM to Conventional Dynamic Contrast- Enhanced and Diffusion-Weighted MRI in Differentiating Benign from Malignant Breast Masses
}

\author{
Qingjun Wang Yong Guo Jing Zhang Zijun Wang Minhua Huang Yun Zhang \\ Department of Radiology, Chinese Navy General Hospital of PLA, Beijing, China
}

\section{Keywords}

Magnetic resonance imaging · Intravoxel incoherent motion · Diffusion-weighted MRI · Dynamic contrastenhanced MRI - Breast cancer

\section{Summary \\ Background: The aim of this study was to determine whether the indicators obtained from intravoxel incoher- ent motion (IVIM) imaging can improve the characteriza- tion of benign and malignant breast masses compared with conventional dynamic contrast-enhanced magnetic resonance imaging (DCE-MRI) and diffusion-weighted magnetic resonance imaging (DW-MRI). Patients and Methods: This study included 23 benign and 31 malig- nant breast masses of 48 patients. Main indicators were initial enhancement ratio (IER), time-signal intensity curve (TIC), apparent diffusion coefficient (ADC), tissue diffusivity (D), pseudodiffusivity $\left(D^{*}\right)$, and perfusion frac- tion (f). The discriminative abilities of the different mod- els were compared by means of receiver operating char- acteristic (ROC) curve and area under the ROC curve (AUC) analysis. Results: D had the highest AUC (0.980), sensitivity (93.55\%), specificity (100\%), and diagnostic accuracy (96.36\%). Both D and TIC could provide the in- dependent predicted features for malignant breast masses. The combination of D and TIC had an AUC of up to 0.990 . Conclusion: D of IVIM can effectively comple- ment existing conventional DCE-MRI and DW-MRI in dif- ferentiating malignant from benign breast masses. IVIM combined with DCE-MRI is a robust means of evaluating breast masses.}

(C) 2016 S. Karger GmbH, Freiburg

\section{Introduction}

In comparison with all other breast magnetic resonance imaging (MRI) techniques, dynamic contrast-enhanced MRI (DCEMRI) and apparent diffusion coefficient (ADC) calculated using diffusion-weighted MRI (DW-MRI) are the most widely used [1, 2]. However, the overlaps of DCE-MRI and ADC in malignant and benign breast lesions indicate that neither is as such the best tool for diagnosing breast lesions [3-6]. Intravoxel incoherent motion (IVIM) imaging has been applied for the characterization of breast cancer and discrimination between breast cancer and benign breast lesions $[7,8]$; however, so far, it has not been shown whether IVIM alone or combined with DCE-MRI or DW-MRI could provide better diagnostic accuracy than DCE-MRI or DW-MRI alone.

The purpose of this study was to retrospectively compare the IVIM parameters obtained with multi-b-values on benign and malignant breast masses and to determine whether these parameters alone or combined with DCE-MRI or DW-MRI can distinguish between malignant and benign breast masses with higher accuracy compared to conventional DCE-MRI and DW-MRI alone or combined models.

\section{Patients and Methods}

Study Population

This retrospective study was approved by our institutional review board. Written consent was obtained from all subjects before scans were performed. A review of our MRI database was performed to identify eligible patients who underwent breast MRI from February 10, 2012 to August 15, 2013.

\section{Inclusion and Exclusion Criteria}

Breast MRI was performed prior to biopsy or surgery. Lesions with any of the following features were excluded from the study: non-mass, non-enhancement, preoperative biopsy, neoadjuvant chemotherapy, and radiation therapy. A total of 23 benign masses ( 21 women) and 31 malignant masses ( 27 women)

\section{KARGER}

() 2016 S. Karger GmbH, Freiburg 
Table 1. Features of indicators for benign and malignant breast masses

\begin{tabular}{|c|c|c|c|}
\hline Indicators $^{\mathrm{a}}$ & $\begin{array}{l}\text { Benign lesions } \\
(\mathrm{n}=23)\end{array}$ & $\begin{array}{l}\text { Malignant lesions } \\
(\mathrm{n}=31)\end{array}$ & $\mathrm{p}$ values \\
\hline \multicolumn{4}{|l|}{ DCE-MRI } \\
\hline IER, \% & $167.98 \pm 45.56$ & $230.09 \pm 34.50$ & $<0.001$ \\
\hline TIC, n (\%) & & & $<0.001$ \\
\hline Type I & $18(78.26)$ & $3(9.68)$ & $<0.001$ \\
\hline Type II & $4(17.39)$ & $6(19.35)$ & 0.854 \\
\hline Type III & $1(4.35)$ & $22(70.97)$ & $<0.001$ \\
\hline \multicolumn{4}{|l|}{ DW-MRI } \\
\hline $\mathrm{ADC}_{0,1000} \leq 1.09(\times 10-3 \mathrm{~mm} 2 / \mathrm{s})$ & $1.22 \pm 0.20$ & $1.03 \pm 0.14$ & $<0.001$ \\
\hline $\mathrm{ADC}_{50,800} \leq 1.10(\times 10-3 \mathrm{~mm} 2 / \mathrm{s})$ & $1.24 \pm 0.21$ & $1.04 \pm 0.15$ & $<0.001$ \\
\hline \multicolumn{4}{|l|}{ IVIM } \\
\hline $\mathrm{D}(\times 10-3 \mathrm{~mm} 2 / \mathrm{s})$ & $1.32 \pm 0.15$ & $0.91 \pm 0.15$ & $<0.001$ \\
\hline $\mathrm{D}^{*}(\times 10-3 \mathrm{~mm} 2 / \mathrm{s})$ & $45.46 \pm 25.25$ & $26.54 \pm 21.91$ & 0.005 \\
\hline $\mathrm{f}, \%$ & $15.16 \pm 9.90$ & $21.18 \pm 7.84$ & 0.016 \\
\hline \multicolumn{4}{|c|}{$\begin{array}{l}\text { aUnless otherwise indicated, continuous data are presented as the mean } \pm \text { standard deviation. } \\
\text { DCE-MRI = Dynamic contrast-enhanced magnetic resonance imaging; IER = initial enhancement ratic } \\
\text { TIC = time-signal intensity curve; DW-MRI = diffusion-weighted magnetic resonance imaging; } \\
\text { ADC = apparent diffusion coefficient; IVIM = intravoxel incoherent motion; D = tissue diffusivity; } \\
D^{*}=\text { pseudodiffusivity; } \mathrm{f}=\text { perfusion fraction. }\end{array}$} \\
\hline
\end{tabular}

were included in the study. The mean age of the patients was $46.85 \pm 8.63$ years. Benign masses included fibroadenoma $(n=22)$ and papilloma $(n=1)$. Malignant masses included invasive ductal carcinoma $(\mathrm{n}=28)$, ductal carcinoma in situ $(\mathrm{n}=1)$, and mixed pathology (invasive ductal carcinoma and ductal carcinoma in situ, $n=2$ ). All 31 malignant masses and 20 of the benign masses were confirmed by surgical pathology. The remaining 3 benign masses were confirmed by ultrasound-guided needle biopsy. A total of 7 lesions were excluded based on the exclusion criteria: 6 non-mass-like enhanced lesions in 5 patients and 1 lesion in a patient with neoadjuvant chemotherapy.

\section{MRI Acquisition}

All MRI examinations were performed with a 3.0T imager (GE Signa HDX GE Healthcare, Milwaukee, WI, USA) by means of a bilateral 8-channel phased array coil (Sentinelle Medical, Toronto, Canada). The images were acquired in axial orientation and in the following order: T2-weighted fast spin echo sequence, T1-weighted non-fat-suppressed sequence, DW sequence, IVIM sequence, and finally 3-dimensional T1-weighted fat-suppressed DCE sequence.

Imaging parameters of DCE-MRI were as follows: repetition time/echo time $=4.1 / 2.1 \mathrm{~ms}$, field of view $=28 \mathrm{~cm}$, nex $=0.71$, matrix $=300 \times 300$, slice thickness $=1.2 \mathrm{~mm}$, gap $=0 \mathrm{~mm}$. The images were obtained with 6 postcontrast acquisitions centered at 40,120,200,280,360, and $440 \mathrm{~s}$. Gadopentetate dimeglumine (Magnevist; Bayer Healthcare, Berlin, Germany) was injected intravenously as a bolus $(0.1 \mathrm{mmol} / \mathrm{kg}$ body weight) by a power injector at $2 \mathrm{ml} / \mathrm{s}$, followed by a $20 \mathrm{ml}$ saline flush.

Imaging parameters of DW-MRI were as follows: frequency-selective fat saturation, parallel acquisition factor $=2.0$, repetition time/echo time $=2,500 / 88.2$ $\mathrm{ms}$, field of view $=28 \mathrm{~cm}$, nex $=2$, matrix $=128 \times 128$, slice thickness $=6.0 \mathrm{~mm}$, gap $=1 \mathrm{~mm}$. b-values were 0 and $1,000 \mathrm{~s} / \mathrm{mm}^{2}$. The scan time was $45 \mathrm{~s}$.

Imaging parameters of IVIM were as follows: frequency-selective fat saturation, parallel acquisition factor $=2.0$, repetition time $/$ echo time $=2,000 / 85 \mathrm{~ms}$, field of view $=28 \mathrm{~cm}$, nex $=4$, matrix $=128 \times 128$, slice thickness $=6 \mathrm{~mm}$, gap $=$ $1 \mathrm{~mm} .10$ b-values were used: $0,10,20,50,100,200,300,400,600,800 \mathrm{~s} / \mathrm{mm}^{2}$. The total scan time was $226 \mathrm{~s}$.

\section{Image Analysis}

All images were transferred to a processing workstation (ADW 4.3; GE Healthcare) and interpreted by 2 radiologists in a consensus-oriented way. Each lesion was assigned to the 2 radiologists who did not know the histopathologic outcomes. The initial enhancement ratio (IER) and time-signal intensity curve
(TIC) were measured and calculated on DCE images. The $\mathrm{ADC}_{0,1000}$ value was derived from the ADC maps of DWI with $b=0$ and $1,000 \mathrm{~s} / \mathrm{mm}^{2}$. Values of $\mathrm{D}$, $\mathrm{D}^{*}$, and $\mathrm{f}$ were acquired on IVIM images. The $\mathrm{ADC}_{50,800}$ value was derived from the ADC maps of DWI with $b=0$ and $800 \mathrm{~s} / \mathrm{mm}^{2}$ calculated from the IVIM images.

According to the DCE images of the 3rd (2nd postcontrast) series, the slice which showed the maximum transverse diameter of a lesion was chosen and a region of interest (ROI) was manually placed on the most enhancing portion of the lesion. The signal intensity (SI) between the 1st (precontrast) and the 3rd DCE series was calculated and referred to as IER (\%). The TIC was categorized into 3 types based on the percentage change of SI between the 3rd (2nd postcontrast) and the 7th (last postcontrast) DCE series with a 5\% cutoff range [9]: Type I, persistent pattern (percentage increase of SI > 5\%); Type II, plateau pattern (deviation of percentage increase of SI between $+5 \%$ and $-5 \%$ ); and Type III, washout pattern (percentage decrease of SI $>5 \%$ ). The DCE ROI was copypasted into the DWI image. The ADC value was acquired as the mean value of voxels in ROI on ADC maps with the formula described in previous reports [10]. For IVIM, 3 diffusivity values were set as follows: the diffusion coefficient of slow or non-perfusion-related molecular diffusion $\left(D, \times 10^{-3} \mathrm{~mm}^{2} / \mathrm{s}\right)$, which represents true molecular diffusion; the diffusion coefficient of fast or perfusion-related diffusion-based diffusion $\left(D^{*}, \times 10^{-3} \mathrm{~mm}^{2} / \mathrm{s}\right)$, which represents perfusion-related diffusion; and the perfusion-related diffusion fraction (f, \%), which represents the fractional volume occupied in the voxel by flowing spins. The calculation of $\mathrm{D}, \mathrm{D}^{*}$, and $\mathrm{f}$ was performed with the research software Multiple ADC (MADC) with the formula described in previous studies [11, 12]. The DCE ROI was also copy-pasted into the IVIM images. The radiologists responsible for measurement would manually adjust the ROI on IVIM images in order to avoid going beyond the outline of the lesion.

\section{Statistical Analysis}

The one-sample Kolmogorov-Smirnov test was first used to analyze continuous variables for normality. Continuous variables with normal distribution were expressed as mean \pm standard deviation (SD). The Student's unpaired $t$ test and $\chi^{2}$ test were applied to estimate the differences in continuous variables and enumeration variables between group pairs. Univariate and multivariate logistic regression models were performed to identify indictors that optimally discriminated malignant from benign lesions, and the diagnostic accuracy of these indicators was evaluated in terms of sensitivity, specificity, and area under the receiver operating characteristic (ROC) curve (AUC). The MR feature with 

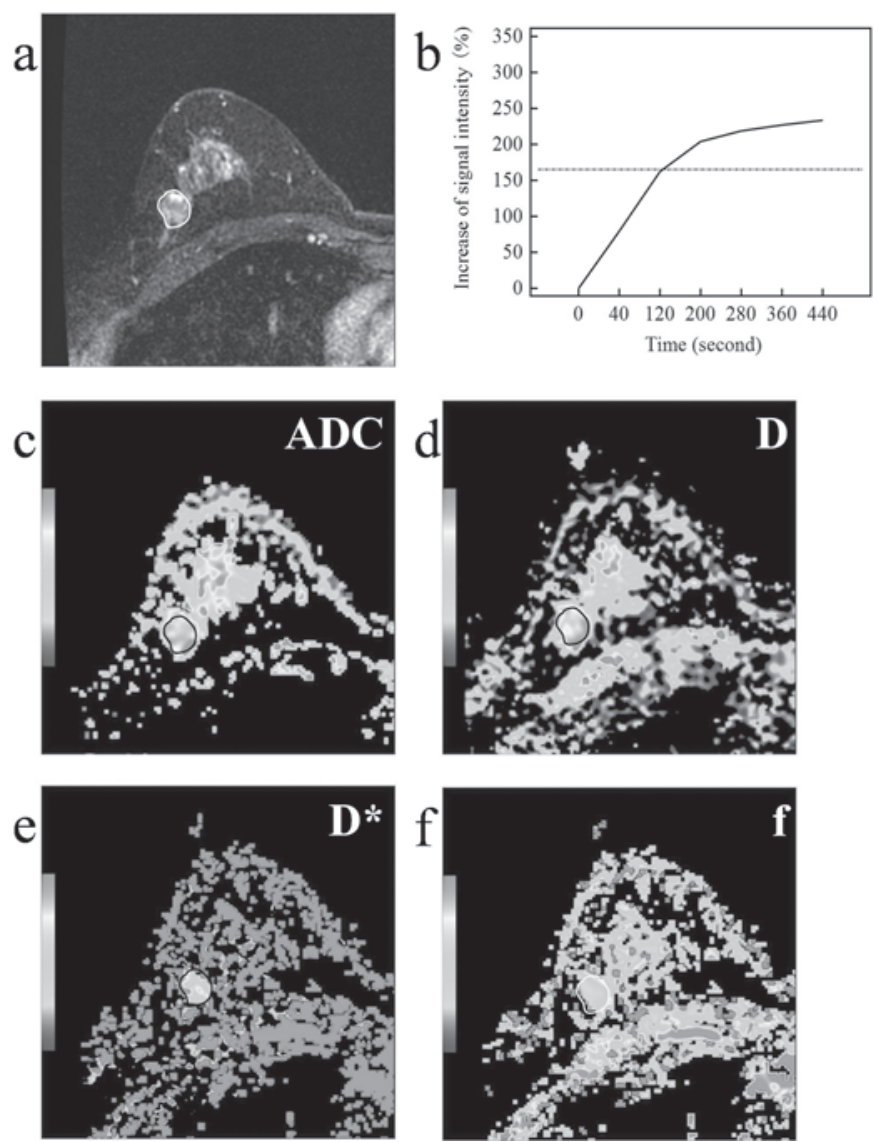

Fig. 1. Fibroadenoma on magnetic resonance images in a 47-year-old woman a Contrast-enhanced magnetic resonance imaging. b Time-signal intensity curve (TIC) Type I. c On diffusion-weighted images, the mean apparent diffusion coefficient $\left(\mathrm{ADC}\right.$ ) was $1.12 \times 10^{-3} \mathrm{~mm}^{2} / \mathrm{s}$. d, e, f On intravoxel incoherent motion (IVIM) images, tissue diffusivity (D), pseudodiffusivity $\left(\mathrm{D}^{*}\right)$, and perfusion fraction (f) were $1.34 \times 10^{-3} \mathrm{~mm}^{2} / \mathrm{s}, 80.80 \times 10^{-3} \mathrm{~mm}^{2} / \mathrm{s}$, and $17.20 \%$, respectively.

the best diagnostic efficacy in each imaging protocol was included in the combined models to evaluate the combined prediction probability for malignancy. 3 months after the 1st measurement, all lesions were reviewed again by another 2 readers to calculate interobserver variability by means of the intraclass correlation coefficient (ICC) (continuous variables) and k-coefficient (enumeration variables). A 2-tailed test pattern was used in all statistical analyses with the level of statistical significance determined as $\mathrm{p}<0.05$. All statistical calculations were performed with SPSS for Windows, version 17.0 (IBM Corp., Armonk, NY, USA). ROC curve analysis was performed using MedCalc software, version 11.4.2.0 (MedCal Software, Mariakerke, Belgium).

\section{Results}

Features of Benign and Malignant Masses on DCE, DW, and IVIM Images

The mean ROI area of benign masses was $87.5 \mathrm{~mm}^{2}$ (range $55.3-189.7 \mathrm{~mm}^{2}$ ). The mean ROI area of malignant masses was $159.9 \mathrm{~mm}^{2}$ (range 82.6-243.2 $\mathrm{mm}^{2}$ ). The results of the comparison between indicators of benign and malignant masses are detailed in table 1. Examples of benign and malignant masses with DCE, DW, and IVIM features are provided in figures 1 and 2.
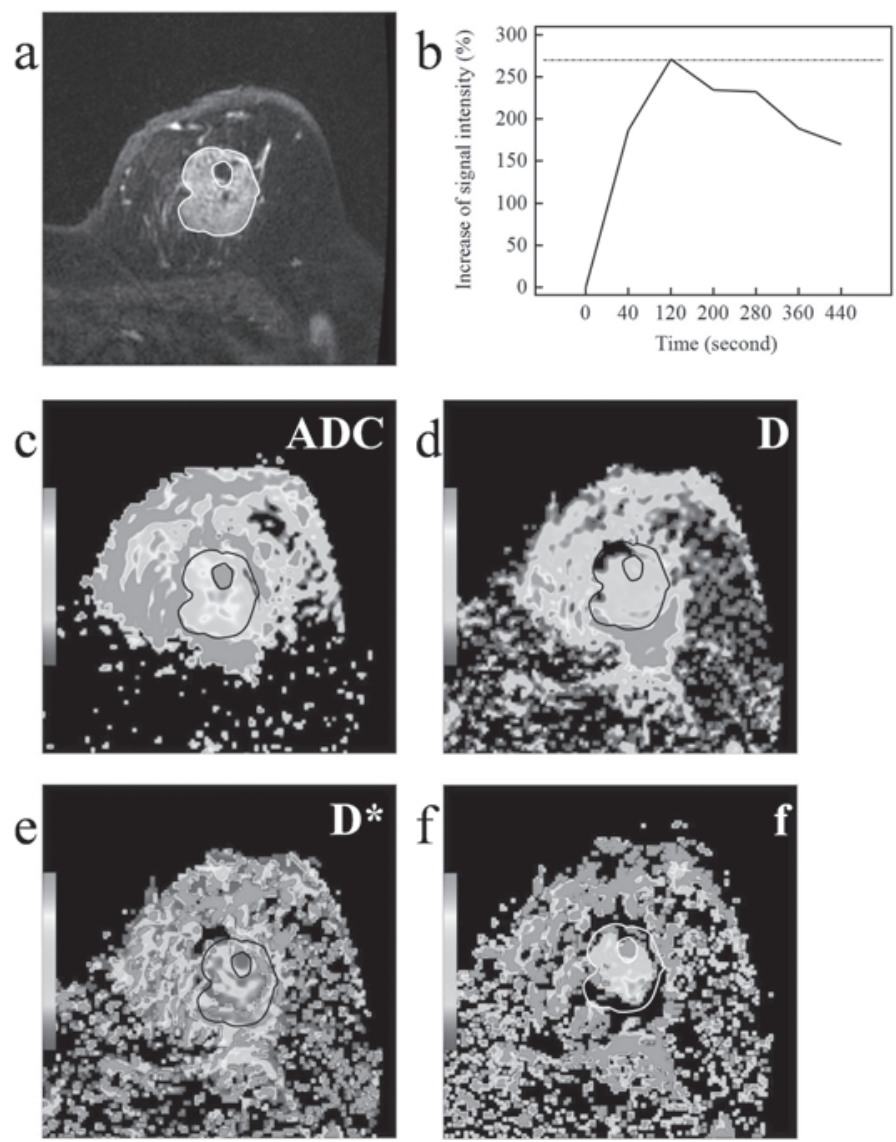

Fig. 2. Invasive ductal carcinoma (grade II) on magnetic resonance images in a 55-year-old woman. a Contrast-enhanced magnetic resonance imaging. b Time-signal intensity curve (TIC) Type III. c On diffusion-weighted images, the mean apparent diffusion coefficient (ADC) was $0.92 \times 10^{-3} \mathrm{~mm}^{2} / \mathrm{s}$. d, e, f On intravoxel incoherent motion (IVIM) images, tissue diffusivity (D), pseudodiffusivity $\left(D^{*}\right)$, and perfusion fraction (f) were $0.85 \times 10^{-3} \mathrm{~mm}^{2} / \mathrm{s}, 17.90 \times 10^{-3}$ $\mathrm{mm}^{2} / \mathrm{s}$, and $25.10 \%$.

Discrimination of Malignant Masses with Univariate Modeling

Univariate logistic regression showed that D, TIC Type II and III, and IER provided the most accurate discrimination between malignant and benign masses, followed by $\mathrm{ADC}_{50,800}, \mathrm{ADC}_{0,1000}$, $\mathrm{D}^{*}$, and $\mathrm{f}$. The individual indicators for discrimination of malignant masses of DEC-MRI, DW-MRI, and IVIM are shown in the online supplementary table (www.karger.com/?DOI=447765).

Discrimination of Malignant Masses with Multivariate Modeling Stepwise multivariate analysis of univariate discriminators (IER, TIC, ADC, D, $\mathrm{D}^{*}$, and $\mathrm{f}$ ) identified D and TIC as the significant independent predictors of malignant masses $(p=0.007$ and 0.038 , respectively). The combined models for discrimination of malignant masses are displayed in the online supplementary table (www.karger. com/?DOI=447765). Figure 3 shows multiple comparisons of ROC curves of individual and combined models. The curves of the 3 combined models were significantly or marginally significantly higher than those of TIC or ADC alone ( $\mathrm{p}=0.004-0.069$ ). However, there was no significant difference between the 3 combined models and no significant difference between $\mathrm{D}$ and the 3 combined models (all $\mathrm{p}>0.05$ ). 


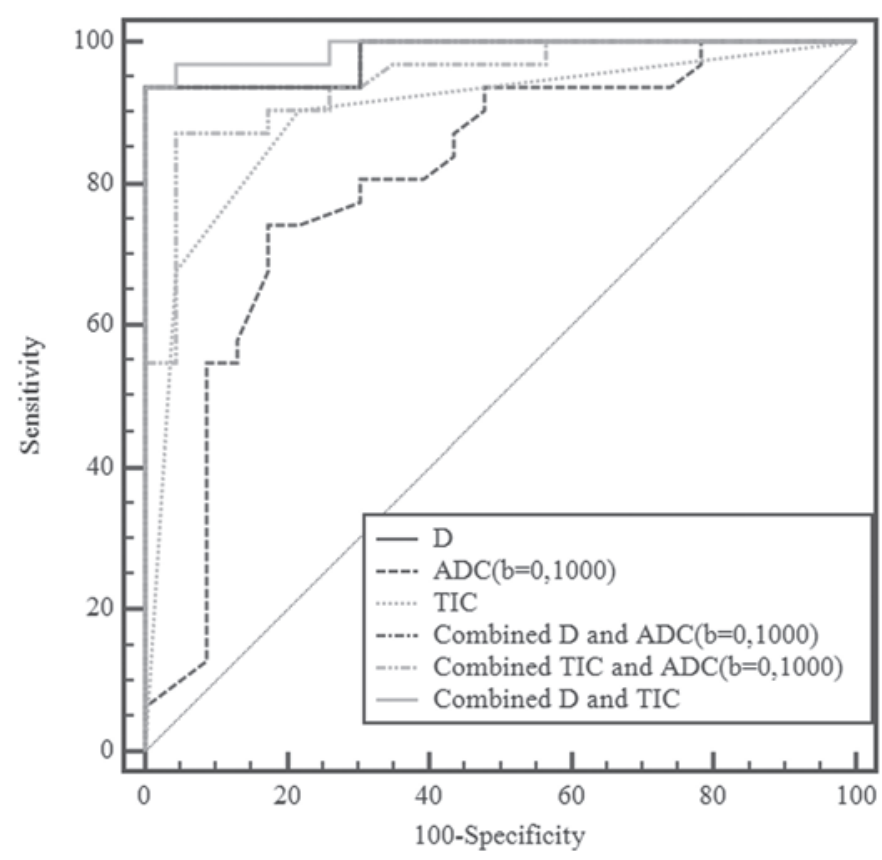

Fig. 3. Pairwise comparisons in receiver operating characteristic (ROC) curves of tissue diffusivity (D), apparent diffusion coefficient $\left(\mathrm{ADC}_{0,1000}\right)$, timesignal intensity curve (TIC), and combined models. The graph shows that the model incorporating both $\mathrm{D}$ and TIC provided the best discriminative ability. The pairwise comparisons in ROC curves of $\mathrm{D}, \mathrm{ADC}_{50,800}$, TIC, and combined models were similar to those of $\mathrm{D}, \mathrm{ADC}_{0,1000}$, TIC, and combined models.

\section{Interobserver Agreement}

There was excellent interobserver agreement in IER with an ICC of 0.917 (95\% confidence interval (CI) 0.859-0.951). For TIC, the intraobserver agreement was relatively good with a k-coefficient of 0.612. There was excellent interobserver agreement for ADC with an ICC of 0.938 (95\% CI 0.893-0.965). There also was excellent interobserver agreement for 2 IVIM parameters: D with an ICC of 0.941 (95\% CI 0.891-0.962) and f with an ICC of 0.806 (95\% CI $0.680-0.886)$. For $\mathrm{D}^{\star}$, the intraobserver agreement was relatively good with an ICC of 0.731 (95\% CI 0.570-0.842).

\section{Discussion}

Our study revealed that D of IVIM has the highest diagnostic accuracy in differentiating malignant from benign breast masses among all the individual imaging indicators. The combination of $\mathrm{D}$ and TIC maximized the AUC to that achievable with other combined models.

Our results showed that compared with conventional ADC of DW-MRI, D significantly improved diagnostic accuracy in the characterization of breast masses. In principle, the histologic characteristics reflected by D of IVIM are similar to those of ADC of DWI; however, D should be superior to ADC because it can demonstrate more precisely the true diffusion without influence of perfusion-related diffusion [13]. Although our results confirmed the superiority of $\mathrm{D}$ over $\mathrm{ADC}$ once again in clinical practice, the extent of this superiority should be further researched given the heterogeneity of ADC in terms of sensitivity and specificity between our study and previous studies [6]. An optimal pair of bvalues used in conventional DW-MRI may be able to decrease the superiority of D over ADC. As with most previous studies [14], we used a pair of 0 and $1,000 \mathrm{~s} / \mathrm{mm}^{2}$ as the b-values in the conventional breast DW-MRI. Some studies indicated that $50 \mathrm{~s} / \mathrm{mm}^{2}$ and 800 or $1,000 \mathrm{~s} / \mathrm{mm}^{2}$ may be the more optimized low and high bvalues in conventional breast DW-MRI on 3.0T MRI [14, 15]. To test whether these potentially optimized b-values (50 and $800 \mathrm{~s} /$ $\mathrm{mm}^{2}$ ) can lead to a better result similar to IVIM compared to our current $b$-values $\left(0\right.$ and $\left.1,000 \mathrm{~s} / \mathrm{mm}^{2}\right)$, images of $b=50$ and $b=800$ $\mathrm{s} / \mathrm{mm}^{2}$ from IVIM were selected to calculate the $\mathrm{ADC}_{50,800}$ maps. Our results showed that both the $\mathrm{ADC}_{0,1000}$ and $\mathrm{ADC}_{50,800}$ were inferior to the $\mathrm{D}$ of IVIM in discriminating malignant from benign breast lesions. However, it should be noted that $\mathrm{ADC}_{50,800}$ presented higher $\mathrm{AUC}$ and specificity values than $\mathrm{ADC}_{0,1000}$, although both had the same sensitivity values. Thus, the b-values 50 and 800 $\mathrm{s} / \mathrm{mm}^{2}$ may be better suited than 0 and $1,000 \mathrm{~s} / \mathrm{mm}^{2}$ in conventional breast DW-MRI on 3.0T MRI.

In the present study, the mean $\mathrm{D}^{*}$ value of benign breast masses was higher than that of malignant masses. Compared with ADC, $\mathrm{D}^{\star}$ cannot further increase the diagnostic sensitivity and specificity. This may be related to the varying tumor vascularity among different masses [7]. In our study, the mean $f$ value of benign breast masses was lower than that of malignant ones, and $\mathrm{f}$ could further increase the diagnostic sensitivity compared with ADC, although the AUC value of $\mathrm{f}$ was the lowest in the present study. Compared with another breast IVIM study by Liu et al. [7], in our study the mean $\mathrm{D}^{*}$ value and $\mathrm{f}$ value for both benign and malignant breast lesions were relatively lower. This may be due to the different quantity and distribution of low and high b-values used in IVIM.

Multiparametric MRI of the breast can provide unique information about breast lesions and therefore has the potential to significantly improve breast cancer diagnosis [2]. In the present study, we assessed the diagnostic efficiency of combined multiparametric data by comparing 3 combined models: D and ADC (both $\mathrm{ADC}_{0,1000}$ and $\mathrm{ADC}_{50,800}$ ), TIC and $\mathrm{ADC}$ (both $\mathrm{ADC}_{0,1000}$ and $\mathrm{ADC}_{50,800}$ ), $\mathrm{D}$ and TIC. Our results showed that there was no significant difference between the 3 combined models, even though the combination of D and TIC maximized the AUC to that achievable with other combined models. However, both sensitivity and specificity of the 3 combined models were significantly or marginally significantly higher than that of TIC or ADC alone, which once again illustrates the importance of multiparametric MRI in breast imaging. Therefore, the combined model of D and TIC has the highest diagnostic value compared with any individual or combined parameters.

Our work has some limitations. Firstly, this was a preliminary and single-center study; besides, the patient samples were of insufficient size. Secondly, the appropriate number of b-values for breast IVIM are still unknown. Considering that the b-value scheme strongly affects the IVIM parameters [16], it should be further evaluated for the most appropriate IVIM in cancer diagnosis. Finally, the histologic distribution of benign and malignant breast 
lesions was narrowed due to the inclusion criteria. The main aim of the present study was to determine whether the IVIM parameters alone or combined with DCE-MRI and DW-MRI can differentiate malignant from benign breast lesions with higher accuracy and to compare the diagnostic value of different multiparametric models. Hence, non-mass-like enhanced breast lesions were not included in our study given that their most important diagnostic features are distribution and internal enhancement pattern but not functional (such as DCE) or molecular (DWI) MRI [13].

In conclusion, according to our study, D of IVIM can effectively complement existing conventional DCE-MRI and DW-MRI in differentiating malignant from benign breast masses. In addition, using D of IVIM in conjunction with DCE-MRI can produce a robust means of evaluating benign and malignant breast masses.

\section{Online Supplementary Table}

Online Supplementary Table. Diagnostic abilities of individual indicators and combined models for benign and malignant breast masses

To access the online supplementary table please refer to $w w w$. karger.com/?DOI=447765.

\section{Disclosure Statement}

The authors declare that they have no conflict of interest.

\section{References}

1 Moon M, Cornfeld D, Weinreb J: Dynamic contrastenhanced breast MR imaging. Magn Reson Imaging Clin N Am 2009; 17:351-362.

2 Pinker K, Bickel H, Helbich TH, Gruber S, Dubsky P, Pluschnig U, Rudas M, Bago-Horvath Z, Weber M, Trattnig S, Bogner W: Combined contrast-enhanced magnetic resonance and diffusion-weighted imaging reading adapted to the 'Breast Imaging Reporting and Data System' for multiparametric 3-t imaging of breast lesions. Eur Radiol 2013;23:1791-1802.

3 Warren RM, Pointon L, Thompson D, Hoff R, Gilbert FJ, Padhani A, Easton D, Lakhani SR, Leach MO; Group UKMRIiBSS: Reading protocol for dynamic contrast-enhanced MR images of the breast: sensitivity and specificity analysis. Radiology 2005;236:779-788.

4 Yabuuchi H, Matsuo Y, Okafuji T, Kamitani T, Soeda H, Setoguchi T, Sakai S, Hatakenaka M, Kubo M, Sadanaga N, Yamamoto H, Honda H: Enhanced mass on contrast-enhanced breast MR imaging: lesion characterization using combination of dynamic contrastenhanced and diffusion-weighted MR images. J Magn Reson Imaging 2008;28:1157-1165.

5 Partridge SC, Rahbar H, Murthy R, Chai X, Kurland BF, DeMartini WB, Lehman CD: Improved diagnostic accuracy of breast MRI through combined apparen diffusion coefficients and dynamic contrast-enhanced kinetics. Magn Reson Med 2011;65:1759-1767.
6 Chen X, Li WL, Zhang YL, Wu Q, Guo YM, Bai ZL: Meta-analysis of quantitative diffusion-weighted MR imaging in the differential diagnosis of breast lesions. BMC Cancer 2010;10:693.

7 Liu C, Liang C, Liu Z, Zhang S, Huang B: Intravoxel incoherent motion (IVIM) in evaluation of breast lesions: comparison with conventional DWI. Eur J Radiol 2013;82:e782-789.

8 Sigmund EE, Cho GY, Kim S, Finn M, Moccaldi M, Jensen JH, Sodickson DK, Goldberg JD, Formenti S, Moy L: Intravoxel incoherent motion imaging of tumor microenvironment in locally advanced breast cancer. Magn Reson Med 2011;65:1437-1447.

9 El Khouli RH, Macura KJ, Jacobs MA, Khalil TH, Kamel IR, Dwyer A, Bluemke DA: Dynamic contrastenhanced MRI of the breast: quantitative method for kinetic curve type assessment. AJR Am J Roentgenol 2009;193:W295-300.

10 Guo Y, Cai YQ, Cai ZL, Gao YG, An NY, Ma L, Mahankali S, Gao JH: Differentiation of clinically benign and malignant breast lesions using diffusion-weighted imaging. J Magn Reson Imaging 2002;16:172-178.

11 Le Bihan D, Breton E, Lallemand D, Grenier P, Cabanis E, Laval-Jeantet M: MR imaging of intravoxel incoherent motions: application to diffusion and perfusion in neurologic disorders. Radiology 1986;161: 401-407.
12 Ichikawa S, Motosugi U, Ichikawa T, Sano K, Morisaka $\mathrm{H}$, Araki T: Intravoxel incoherent motion imaging of focal hepatic lesions. J Magn Reson Imaging 2013;37: 1371-1376.

13 Tamura T, Usui S, Murakami S, Arihiro K, Fujimoto T, Yamada T, Naito K, Akiyama M: Comparisons of multi b-value DWI signal analysis with pathological specimen of breast cancer. Magn Reson Med 2012;68 890-897.

14 Vermoolen MA, Kwee TC, Nievelstein RA: Apparent diffusion coefficient measurements in the differentiation between benign and malignant lesions: a systematic review. Insights Imaging 2012;3:395-409.

15 Nogueira L, Brandao S, Matos E, Nunes RG, Loureiro J, Ferreira HA, Ramos I: Diffusion-weighted imaging: determination of the best pair of b-values to discriminate breast lesions. Br J Radiol 2014;87:20130807.

16 Pang Y, Turkbey B, Bernardo M, Kruecker J, Kadoury S, Merino MJ, Wood BJ, Pinto PA, Choyke PL: Intravoxel incoherent motion MR imaging for prostate cancer: an evaluation of perfusion fraction and diffusion coefficient derived from different b-value combinations. Magn Reson Med 2013;69:553-562. 